\title{
Adhesion on Nanoorganized Multilayers
}

\author{
Yolla Kazzi ${ }^{1}$ and Houssein Awada ${ }^{2}$ \\ ${ }^{1}$ Faculty of Sciences, Lebanese University, Hadath, P.O. Box 13-5789, Beirut, Lebanon \\ ${ }^{2}$ Centre de Recherche sur les Matériaux Lignocellulosiques (CRML), Université du Québec à Trois-Rivières, \\ Boulevard des Forges, Trois-Rivières, QC, Canada G9A 5 H7
}

Correspondence should be addressed to Yolla Kazzi, ykazzi@ul.edu.lb

Received 10 May 2011; Accepted 25 August 2011

Academic Editor: Jan Skov Pedersen

Copyright ( $\odot 2011$ Y. Kazzi and H. Awada. This is an open access article distributed under the Creative Commons Attribution License, which permits unrestricted use, distribution, and reproduction in any medium, provided the original work is properly cited.

\begin{abstract}
Nanostructured multilayers composed of alternate organic (alkyldithiol) and metallic (gold) layers are grafted onto glass plates and prepared in order to modify the mechanical and local dissipative properties of a thin surface layer of the substrate. The adhesion phenomenon between a polyisoprene elastomer and these layers is presented and verified by two theories, namely, Johnson, Kendall, Roberts (JKR) and linear elastic fracture mechanics. The increase in adhesion with contact time following a power law has been clearly noted.
\end{abstract}

\section{Introduction}

The adhesive failure energy of adhesive joints formed at a low contact pressure during a short contact time is called "tack strength" [1]. Tack represents a limited case of adhesion. It is a property which reflects the ability of a material to adhere instantaneously to a rigid surface, under a low pressure carried out for a short time [2].

Tack is of considerable interest in the field of adhesives and bonding in particular for pressure-sensitive adhesives (PSAs) such as adhesive labels [3].

The use of adhesives is becoming more frequent and their use on several systems requires an in depth understanding of the mechanisms governing their properties.

To date, the respective influences on the adhesion of interfacial interactions and phenomena of local dissipation energy, immediately close to the interface, have not yet been fully established. Interfacial interactions and the dissipation energy both in the volume of materials and near the interface determine, to a large extent, the adhesion energy between two solids in contact.

In the present study, we try to focus on this local dissipation at the interface not in the bulk of the material.

To achieve our goal, thin multilayers, alternatively organic (alkyldithiol, PDMS) and metallic (gold), are deposited on glass plates in order to modify the mechanical properties and local dissipation on the substrate surface.
An adhesion promotor (organosilanes terminated thiol) is first grafted onto the hydrophilic substrate, allowing the deposit of a gold layer by thermal evaporation.

Successive layers of alkyldithiol (or polydimethylesiloxane) and gold can be carried out.

The external face is either gold-or alkyldithiol-terminated methyl allowing the study of the surfaces of different thermodynamic characteristics (Figure 1).

The chemical and structural characterization of these self-assembled layers as well as the study of their adhesive properties in short time (tack) towards a polyisoprene elastomer were performed in the previously published articles $[4,5]$.

The principal objective of this work is to apply an appropriate micromechanical approach such as Johnson, Kendall, Roberts (JKR) and more typically, linear elastic fracture mechanics, in order to be able to correctly interpret the experimental results obtained by using the experimental apparatus developed in the laboratory.

Finally, we discuss the influence of the contact time on energy of adhesion of these numerous layers used towards polyisoprene elastomer.

\section{Materials and Methods}

2.1. Preparation of Nanostructured Multilayers. The multilayers are prepared in the following way [6]. After ultrasonic 


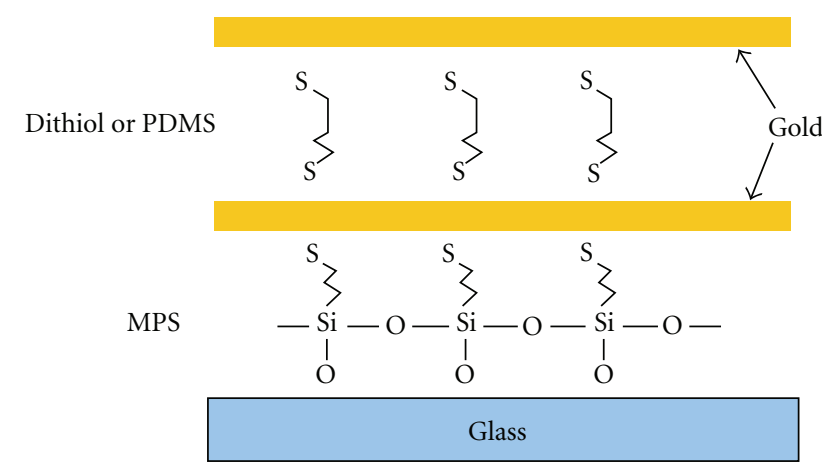

FIGURE 1: Schematic representation of the nanostructured multilayers. The scale between molecules and gold layers is not proportional.

cleaning in chloroform, the surface of a glass plate is hydroxylated by dipping it into a piranha solution $\left(30 \% \mathrm{H}_{2} \mathrm{O}_{2} / 70 \%\right.$ $\mathrm{H}_{2} \mathrm{SO}_{4}$ ) at $50^{\circ} \mathrm{C}$ for $30 \mathrm{~min}$. The glass plate is then rinsed with doubly distilled water and dried under a nitrogen gas jet.

For the first layer of gold, first we immersed glass slides $(18 \mathrm{~h})$ in $1 \mathrm{~mol} \cdot \mathrm{L}^{-1}$ of 3 -mercaptopropyltrimethoxysilane (MPS, 95\%) solution (in toluene). MPS (supplied by ABCR, Karlsruhe-Germany) was used as a coupling agent between glass slides and gold coatings [7, 8]. Second, grafted glass was rinsed with toluene and dried with nitrogen blanket. Finally, the first gold (99.99\% from Blazers) layer was coated under vacuum $\left(10^{-7} \mathrm{mbar}\right)$ using an Edwards 306 evaporator. The thickness of gold layers varied between 2 to $20 \mathrm{~nm}$ approximately.

For the second layer of gold, we have used two kinds of coupling agents, an alkythiol with thio-sulfur group at both ends and a polydimethylsiloxane (PDMS) of molecular weight of $1000 \mathrm{~g} \cdot \mathrm{mol}^{-1}$ and $3000 \mathrm{~g} \cdot \mathrm{mol}^{-1}$, respectively, called PDMS ${ }_{n=10}$ and $\mathrm{PDMS}_{n=37}$. The PDMS has amine end groups on both chain ends.

The sulfur and the amine are able to create a specific and strange link with the gold surface. In both cases, we immersed the substrates in a $1 \mathrm{mmol} \cdot \mathrm{L}^{-1}$ solution of either 1-9 nonanedithiol (95\%, Aldrich) or PDMS $_{n=10}$ (from ABCR, Karlsruhe-Germany) in ethanol and $1 \mathrm{mmol} \cdot \mathrm{L}^{-1}$ solution of $\mathrm{PDMS}_{n=37}$ (from ABCR, Karlsruhe-Germany) in cyclohexane for 18 hours, then the substrates are rinsed with ethanol (in the case of alkylthiol, $\mathrm{PDMS}_{n=10}$ ) and with cyclohexane (in the case of $\mathrm{PDMS}_{n=37}$ ) and dried with nitrogen blanket.

The schematic structure of the multilayers, prepared in this study, is shown in Figure 1.

2.2. Adhesion (Tack) Test. To evaluate tack for the systems glass/multilayers/elastomer, the Johnson, Kendall, Roberts (JKR) test [9] was used. We have chosen the contact between a hemisphere of cross-linked polyisoprene and a plane substrate on which nanostructured multilayers are grafted. With this particular geometry, bulk dissipations are greatly reduced and the contact area can be easily measured. In our study, the polyisoprene is partially cross-linked with dicumyl of peroxide [10]. With the apparatus described in

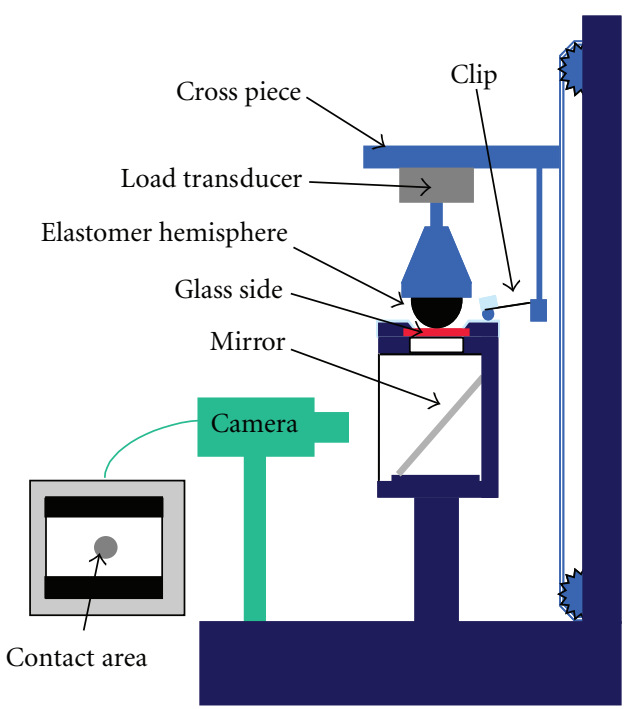

FIgURE 2: Test experimental setup.

Figure 2, the impact and separation rates $\left(10 \mathrm{~mm} \cdot \mathrm{min}^{-1}\right)$ as well as the contact time can be controlled. A high-speed camera (250 image $\cdot \mathrm{s}^{-1}$ ) is used to follow the variation of the contact area between the elastomer and the substrate. Thus, displacements, applied force, and contact area can be measured simultaneously.

The total energy, $G_{\text {tot }}$, needed to break the junction is obtained by integration of positive values of the force $F$ versus displacement:

$$
G_{\mathrm{tot}}=\int_{F \geq 0} F \cdot d z
$$

\section{Results and Discussion}

3.1. Verification of the Validity of JKR and Linear Elastic Fracture Mechanics Theories. We wanted to check if our results were well represented during the separation step by a single relationship between the rate of energy release $G$ and the speed of propagation of the fracture " $v$ ". Such variation reflects a factor of energy dissipation. $a(t)$

The opening speed of the contact is calculated by deriving

$$
V=-\frac{d a}{d t} \cong \frac{a\left(t+d t_{1}\right)-a\left(t-d t_{2}\right)}{d t_{1}+d t_{2}},
$$

where $a=$ contact area.

In order to calculate the rate of the mechanical energy, we adopted for two theories: JKR and the elastic mechanics of fracture, which were applied to our system during the decompression stage.

3.1.1. JKR Theory [9]. The release rate of elastic energy $G$ as a function of the propagation speed " $v$ " is calculated from the values of the applied force $P$ (Newton) and $a$ recorded at time $t$

$$
G=\frac{\left(P-K a^{3} / R\right)^{2}}{6 \pi K a^{3}},
$$




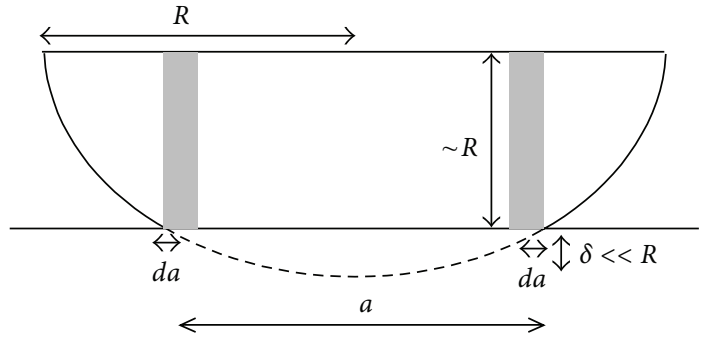

FIgURE 3: Contact hemisphere/substrate.

with $K$ stiffness of the hemisphere and $R$ radius of the hemisphere.

3.1.2. Theory of Linear Fracture Mechanics. In general, when a material is subjected to stresses, it stores elastic energy in the distended and deformed bonds. When we remove the stresses, these bonds return to their positions of equilibrium and consequently release a certain amount of elastic energy. We will now consider what happens when a fracture propagates at the interface between two materials in contact.

Consider Figure 3 which shows a hemisphere (PI) in contact with the substrate (glass). Given that this contact does not affect the whole amount of the hemisphere surface, we consider a cylindrical tube of length $R$ with $R \gg \delta$ ( $\delta$ is the penetration of the hemisphere) and thickness da.

In this case, we consider the energy stored in the tubular volume to be equal to the energy required to propagate the interfacial fracture of a distance da (crown) during separation.

Then, the elastic energy accumulated in the tubular volume $(V)$ is determined by the following relation:

$$
W_{\mathrm{el}}=\frac{1}{2} \sigma \varepsilon V .
$$

Since the behavior of the material is purely elastic, we apply Hooke's law $(\sigma=1 / 2 E \varepsilon)$.

The expression of elastic energy released is then

$$
W_{\mathrm{el}}=\frac{\sigma^{2}}{E} V .
$$
obtain

Knowing that $\sigma=F / S=F / \pi a^{2}$ and $V=2 \pi a d a R$, we

$$
W_{\mathrm{el}}=\frac{2 F^{2}}{\pi a^{3} E} R d a,
$$

where: $F$ is the applied force and $E$ is The elastic modulus.

Moreover, the energy required to propagate the interfacial fracture of " $d a$ " is given by the following relation:

$$
W_{f}=G 2 \pi a d a,
$$

where $G$ is the interfacial fracture energy.

As $W_{\mathrm{el}}=W_{f}$, we obtain

$$
2 \pi a G d a=\frac{2 F^{2}}{\pi a^{3} E} R d a,
$$

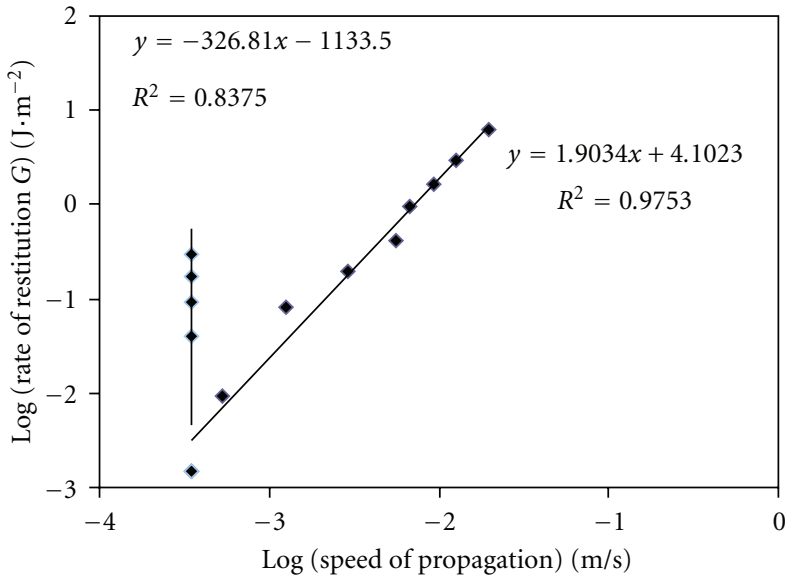

(a)

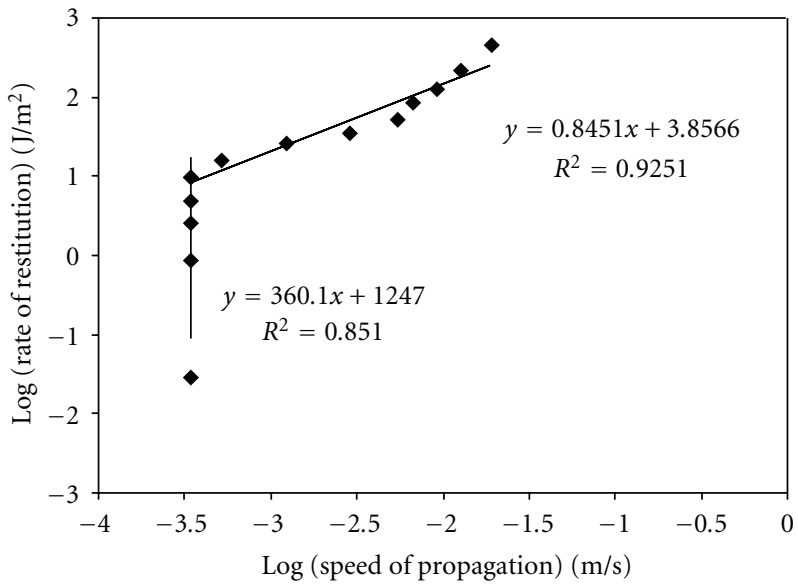

(b)

FIGURE 4: Evolution of fracture energy, $G$, with the rate of fracture " $v$ " (system: IR/Glass/MPS/Gold (3.8 nm)), (a) analysis JKR, (b) linear fracture mechanics.

where finally

$$
G=\frac{F^{2} R}{\pi^{2} a^{4} E} .
$$

The analysis focuses on experimental systems consisting of a compressed hemisphere of IR $(R=19 \mathrm{~mm})$ on a glass substrate supporting nanostructured multilayers during $0.1 \mathrm{~s}$ with a loading force of $0.01 \mathrm{~N}$. They were separated using a withdrawal rate of $10 \mathrm{~mm} \cdot \mathrm{min}^{-1}$.

Figures 4(a) and 4(b) represent, respectively, the variations of $G$ in function of the rate of the fracture " $V$ " according to the JKR theory and to the linear elastic fracture mechanics. In both, Figures 4(a) and 4(b), we used a contact between the hemisphere (IR) and a glass/MPS/gold $(3.8 \mathrm{~nm})$.

Curve (a) is characterized by a decreasing phase until its value of $G$ is equal to $1.53 \cdot 10^{-3} \mathrm{~J} \cdot \mathrm{m}^{-2}$ beyond which the curve shows a linear increasing phase.

This behavior differs substantially from that of Curve (b) which shows increasing phase until a critical value of $G$ is equal to $G=9.91 \mathrm{~J} \cdot \mathrm{m}^{-2}$. Beyond this value, there is also an increasing linear phase. 
TABLE 1: Value of the exponent " $n$ " and coefficient of linear correlation $R^{2}$.

\begin{tabular}{lcc}
\hline Samples & $\begin{array}{c}\text { JKR Theory } \\
\text { Slope of the line }(n)\end{array}$ & $\begin{array}{c}\text { Linear elastic fracture } \\
\text { Mechanics theory } \\
\text { Slope of the line }(n)\end{array}$ \\
\hline Glass/MPS/Gold $(2.9 \mathrm{~nm})$ & 1.8 & 0.7 \\
Glass/MPS/Gold $(5.30 \mathrm{~nm})$ & 1.5 & 0.7 \\
Glass/MPS/Gold $(10.4 \mathrm{~nm})$ & 2.5 & 1 \\
Glass/MPS/Gold $(5.4 \mathrm{~nm}) / \mathrm{Dithiol} /$ gold $(5.85 \mathrm{~nm})$ & 4.9 & 1.3 \\
Glass/MPS/Gold $(5.85 \mathrm{~nm}) / \mathrm{PDMS}_{n=37} / \mathrm{Gold}(5.25 \mathrm{~nm})$ & 3 & 1.5 \\
Glass/MPS/Gold $(5.7 \mathrm{~nm}) / \mathrm{PDMS}_{n=10} /$ Gold $(5.4 \mathrm{~nm})$ & 4.8 & 2 \\
Glass/MPS/Gold $(4.8 \mathrm{~nm}) /$ Dithiol/Gold $(4.8 \mathrm{~nm}) / \mathrm{Hexadecanethiol}$ & 6.8 & 2.6 \\
Glass/MPS/Gold $(4.8 \mathrm{~nm}) /$ Dithiol/Gold $(4.8 \mathrm{~nm}) /$ Hexanethiol & 5.3 & 2.5 \\
Glass/MPS/Gold $(4.8 \mathrm{~nm}) /$ Hexadecanethiol & 9.3 & 1.8 \\
Glass/MPS/Gold $(5.85 \mathrm{~nm}) /$ Hexanethiol & 7.2 & 2.3 \\
\hline
\end{tabular}

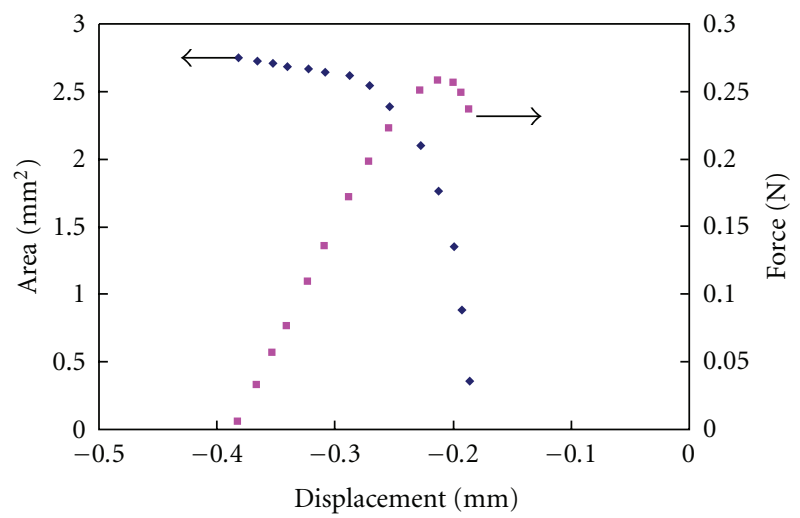

Figure 5: Contact area and displacement force during separation for the contact IR/Glass+MPS+Gold $(3.9 \mathrm{~nm})$.

In both cases, the linear phase corresponds first to a slow propagation of the fracture (little change of the contact area) before an acceleration until complete separation (Figure 5).

Therefore, the basic relation $G=f\left(v^{n}\right)$ with " $n$ " only used to adjust the results from each of these two theories does not apply during the separation step. However, Figure 4 shows that beyond low speed, it is difficult to quantify (field setting regime somehow). We have to use power laws to describe the experimental results correctly.

In our study, we are interested particularly in the linear part of the two curves to represent the evolution of the release rate $G$ as a function of the separation speed for the different substrates analyzed. Table 1 shows that fracture energy $G$ can be expressed at a first approximation as $G \cong v^{n}$.

The values of " $n$ " from the JKR theory are systematically higher than those obtained by the linear fracture mechanics.

The power laws correspond to an exponent " $n$ " between 0.7 and 2.6 for the linear fracture mechanics but between 1.5 and 9.3 for the JKR theory according to the different samples analyzed.

Despite this difference in the values of " $n$ ", the two methods lead to the same trends and confirm a change in the fracture energy $G$ as a function of the speed of separation as a power law type $G \cong v^{n}$.

These differences can be explained by the fact that JKR theory cannot be used here because this theory applies to the equilibrium state, that is, that each variation of force or displacement must be followed by a relaxation phase. In our case, the system is never at equilibrium. We continuously decompress until detachment of the elastomer from the glass plate. We then measure the evolution of the area and force versus time (without allowing total relaxation).

The JKR approach is, therefore, valid at equilibrium. While tack is always determined by choice and necessity, no equilibrium tack is by nature a nonequilibrium quantity.

Finally, we can already note that the exponent " $n$ " ( $n \approx 0.7$ ) obtained with the fracture mechanics for the sample glass/MPS/gold $(5.3 \mathrm{~nm})$ is in concordance with the literature value $(n \approx 0.6)$.

3.2. Influence on Contact Time. We have analyzed the influence of contact time on tack measurement; the load and separation rates are equal to $10 \mathrm{~mm} \cdot \mathrm{min}^{-1}$, and a constant displacement is imposed $(0.1 \mathrm{~mm})$. The contact time between the hemisphere and the grafted glass varies from $0.1 \mathrm{~s}$ to $10000 \mathrm{~s}$. Variations of tack as a function of contact time are given in a bi-logarithmic scale for all systems in Figure 6. Generally the adhesion energy between two solids increases when the contact time increases until an equilibrium state is reached.

This evolution may be due to physicochemical interaction modification at the interface inducing an increase in energy required to separate two surfaces. In all cases, an increasing linear variation is obtained.

We note that the slopes of these variations are similar for all multiple layers with bases of thiol and dithiol. The variations for layers containing PDMS are faster and seem to meet the previous values corresponding to thiol and dithiol systems after a long time (1000 s).

In all cases, tack energy can be written in the form of a power law of the contact time $(t) E g \cong t^{\beta}$ (Figure 6). 
TABLE 2: Values of exponents $\beta$ (slope the straight line) and of the linear correlation coefficient $R^{2}$.

\begin{tabular}{|c|c|c|}
\hline Substrates & Exponents $\beta$ & $\begin{array}{c}\text { Correlation } \\
\text { coefficient } R^{2}\end{array}$ \\
\hline Glass/MPS/Gold (5.1 nm) & 0.04 & 0.99 \\
\hline Glass/MPS/Gold (21.2 nm) & 0.065 & 0.97 \\
\hline Glass/MPS/Gold/Dithiol/Gold (4.8 nm) & 0.072 & 0.99 \\
\hline Glass/MPS/Gold/PDMS $_{n=37} /$ Gold $(5.4 \mathrm{~nm})$ & 0.19 & 0.97 \\
\hline Glass/MPS/Gold/PDMS ${ }_{n=10} /$ Gold $(5.25 \mathrm{~nm})$ & 0.16 & 0.96 \\
\hline Glass/MPS/Gold/Dithiol/Gold/Hexadecanethiol & 0.11 & 0.99 \\
\hline
\end{tabular}

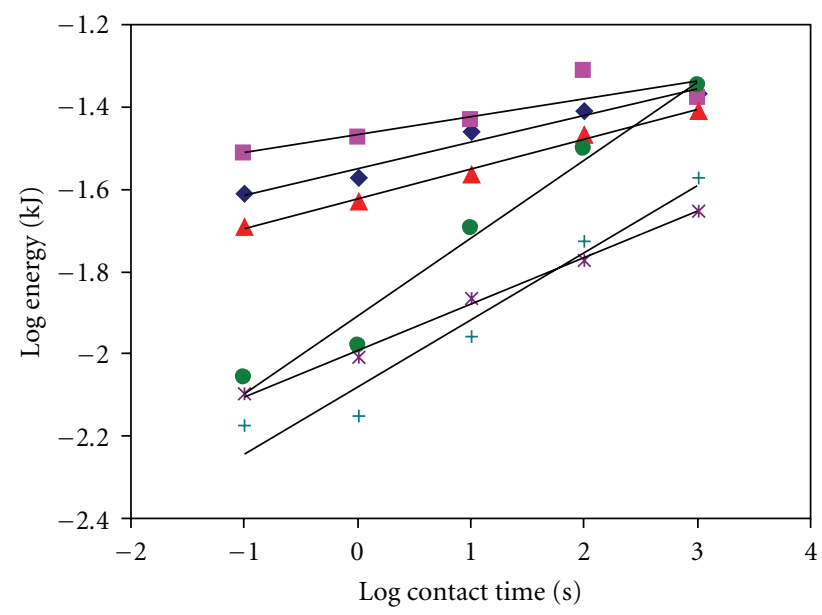

- Glass/MPS/Gold (21.2 nm)

- Glass/MPS/Gold (5.1 nm)

A Glass/MPS/Gold (4.95 nm)/Dithiol/Gold (4.8 nm)

- Glass/MPS/Gold (5.1 nm)/PDMS ${ }_{n=37} /$ Gold $(5.4 \mathrm{~nm})$

* Glass/MPS/Gold (4.8 nm)/Dithiol/Gold (4.95 nm)/Hexadecanethiol

+ Glass/MPS/Gold $(5.25 \mathrm{~nm}) / \mathrm{PDMS}_{n=10} /$ Gold $(5.25 \mathrm{~nm})$

FIGURE 6: Evolution of adhesion energy as a function of time for the different systems Elastomer/Glass grafted (Test JKR: $V_{\text {withdraw }}=$ $\left.10 \mathrm{~mm} \cdot \mathrm{min}^{-1}\right)$.

The values of $\beta$ and correlation coefficient $R^{2}$ are given in Table 2 for different systems.

These results show clearly the dependence of $\beta$ on a gold layer structure and the nature of functional groups at the surface. Indeed, the grafted substrates terminating in one gold layer, where the thickness varies, almost give identical $\beta$ exponents. The representation of total energy of adhesion, as a function of contact time corresponds to two parallel straight lines.

This parallel can be explained by a similar evolution of interfacial interaction in time, moreover, with everything being equal, particularly the dissipation of energy.

Nevertheless, the adherence of a $5 \mathrm{~nm}$ gold layer is always higher than that of $21.1 \mathrm{~nm}$.

This result is assigned to a wormlike disjointed, connected gold structure, which might influence fracture propagation.

Concerning the system with two gold layers separated by PDMS, with alkylthiol terminated or not, the energy of adhesion evolves in time faster than in the case of the same system with dithiols. First of all, exponent $\beta$ values for this sample (Table 2) are still lower than the results obtained in the case of interdiffusion $(\beta=0.5)[11,12]$.

The adhesion energy evolution with time is not due to interdiffusion of grafts; PDMS or hexadecanethiol in polyisoprene (IR) $\beta$ values found are close to those obtained by Zosel and Granvill [13]. At longer times (more than $1 \mathrm{~s}$ ), the increase of the adhesion energy is proportional to $\mathrm{tc}^{0.1}$ for polyisobutylene and tc $\mathrm{tc}^{0.2}$ for polybutylacrylate.

The improvement in adherence with time can probably be assigned to the relaxation of stress caused by a rigid surface of substrates on the elastomer, making better contact until complete wetting after about $1000 \mathrm{~s}$. Therefore, this explains the increase in the contact area, at a microscopic level. The contact area develops by relaxation of the elastomer before separation leading to better tack properties, because the measured force is directly proportional to the real contact area, with everything else being equal.

However, the hypothesis of the wetting phenomenon is not the only explanation for the power function increase in the total energy with the contact time. We can also mention a molecular reorganization at the interface affecting the localization and concentrations of molecules (polymer, alkylthiols, and PDMS molecules) at the interface. After a long time, we finally find sufficient polymer chains adsorbed at the surface leading to a high energy of separation.

\section{Conclusion}

In this study, we investigate the adhesion between a hemisphere of IR and nanoorganised substrate. We can get two major pieces information from our experimental results.

(1) The application of both theories JKR and the linear elastic fracture mechanics, showing that the relationship between the rate of release energy $G$ and the speed of propagation of the fracture " $V$ ". $G=$ $f\left(v^{n}\right)$ with single " $n$ " is not applicable during all the separation steps.

(2) An increase in global energy with contact time until it reaches an equilibrium level. This phenomenon may be due two reasons. The first is a modification of the interactions at the interface caused by a competition of localization of molecules at the interface polyisoprene/grafted glass. The second is a relaxation over time of stress exerted on the elastomer. 


\section{References}

[1] A. Zosel, "Adhesion and tack of polymers: influence of mechanical properties and surface tensions," Colloid \& Polymer Science, vol. 263, no. 7, pp. 541-553, 1985.

[2] F. H. Wetzel, "The Characterization of pressure sensitive adhesives," ASTM Bulletin, vol. 221, pp. 64-68, 1957, ASTM D 1878-61T.

[3] A. Agirre, J. Nase, E. Degrandi, C. Costantino, and J. M. Asua, "Improving adhesion of acrylic waterborne PSAs to low surface energy materials: introduction of stearyl acrylate," Journal of Polymer Science A, vol. 48, no. 22, pp. 5030-5039, 2010.

[4] Y. Kazzi, H. Awada, M. O. David, and M. Nardin, "Characterization of nano-organized multilayers constituted by successive inorganic (gold) and organic (alkylthiols) layers," Surface and Interface Analysis, vol. 39, no. 8, pp. 691-697, 2007.

[5] Y. Kazzi, H. Awada, and M. Nardin, "Adhesion on nanoorganized multilayers: surface thermodynamics and local energy dissipation," Advances in Physical Chemistry, vol. 2010, 11 pages, 2010 .

[6] M. O. David, T. Gerriet, M. Nardin, and J. Schultz, "Nanostructured multilayers and their influence on tack of crosslinked elastomers," Langmuir, vol. 16, no. 18, pp. 7346-7350, 2000.

[7] H. Awada, G. Castelein, and M. Brogly, "Use of chemically modified AFM tips as a powerful tool for the determination of surface energy of functionalised surfaces," Surface and Interface Analysis, vol. 124, pp. 129-134, 2005.

[8] H. Awada, G. Castelein, and M. Brogly, "Quantitative determination of surface energy using atomic force microscopy: the case of hydrophobic/hydrophobic contact and hydrophilic/ hydrophilic contact," Surface and Interface Analysis, vol. 37, no. 9, pp. 755-764, 2005.

[9] K. L. Johnson, K. Kendall, and A. D. Roberts, "Surface energy and the contact of elastic solids," Proceedings of the Royal Society A, vol. 324, pp. 301-313, 1971.

[10] M. O. David, T. Nipithakul, M. Nardin, J. Schultz, and K. Suchiva, "Influence of nonrubber constituents on tack of natural rubber. I. At very short times of contact (pendulum test)," Journal of Applied Polymer Science, vol. 78, no. 8, pp. 14861494, 2000.

[11] P. G. De Gennes, "Reptation of a polymer chain in the presence of fixed obstacles," The Journal of Chemical Physics, vol. 55, pp. 572-579, 1971.

[12] M. Prager and M. tirell, "The healing process at polymerpolymer interfaces," The Journal of Chemical Physics, vol. 75, pp. 5194-5198, 1981.

[13] A. Zosel, "The tack of polymer and its relation to molecular structure," in Proceedings of the European Adhesion Conference (EURADH '96), N.W. Granvill, Ed., pp. 47-51, 1996. 


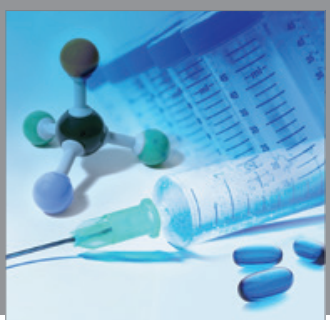

International Journal of

Medicinal Chemistry

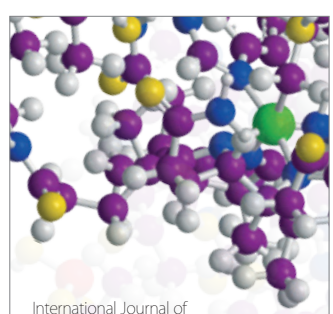

Carbohydrate Chemistry

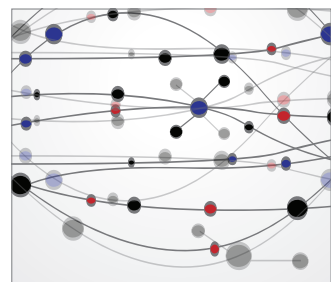

The Scientific World Journal
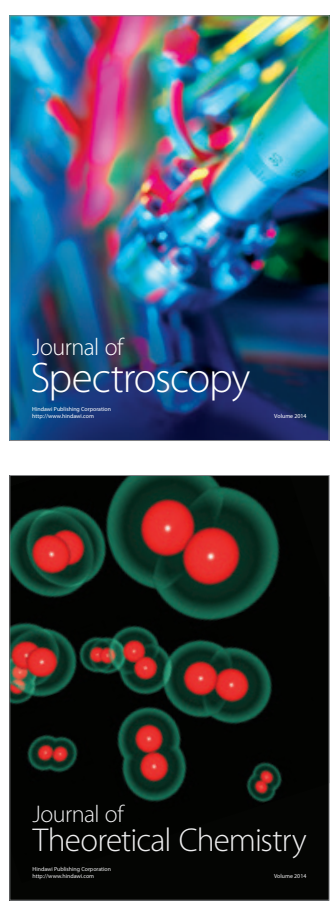
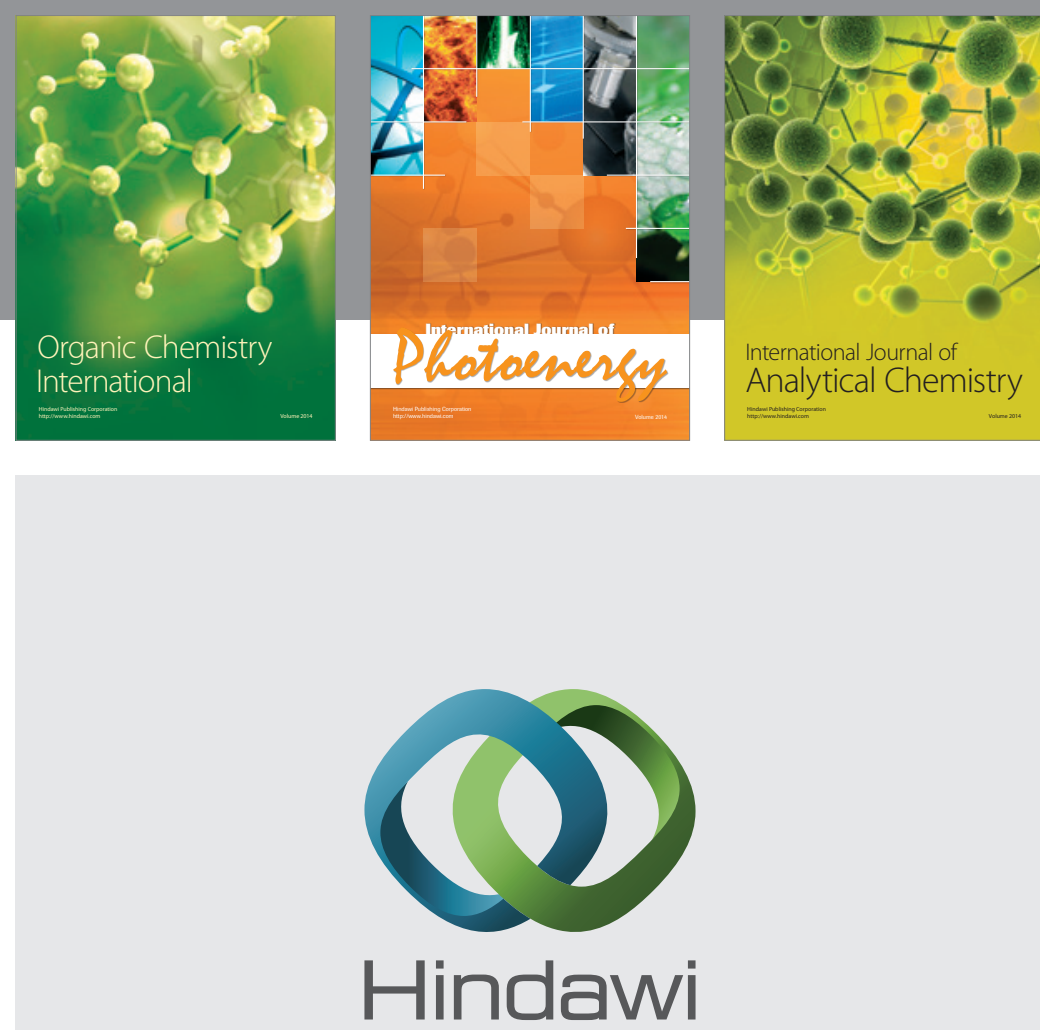

Submit your manuscripts at

http://www.hindawi.com
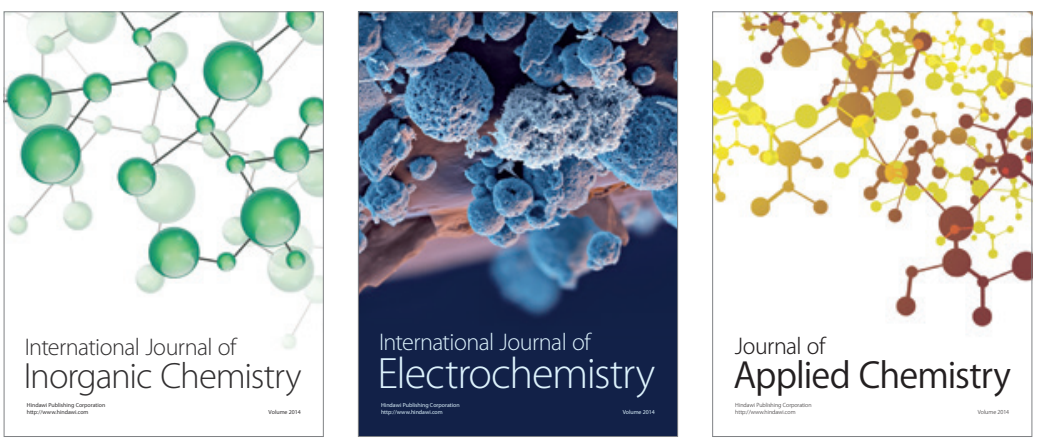

Journal of

Applied Chemistry
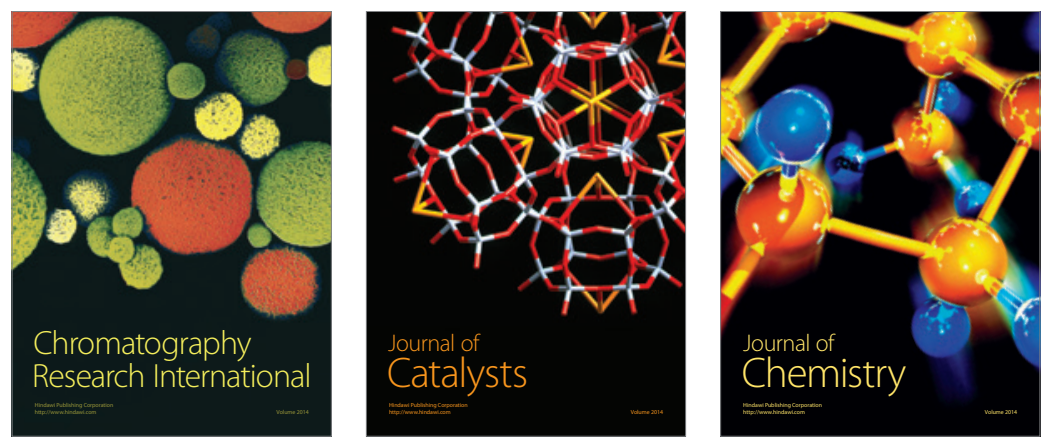
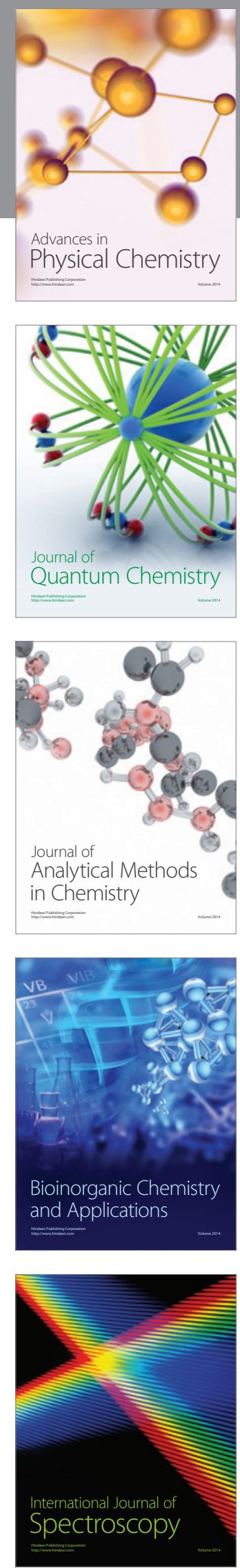